\title{
Effect of Organogelator Concentration on Gelation Kinetics and Drug Release Behaviour of Paracetamol-loaded Soybean Oleogels
}

\author{
Dipanjana Ash a, Sutapa Biswas Majee ${ }^{b}$, Gopa Roy Biswas ${ }^{c}$ \\ a. Department of Pharmaceutics, BCDA College of Pharmacy \& Technology, 78/1 Jessore Road (S), Hridaypur, Barasat, Kolkata, West Bengal, India. \\ b Department of Pharmacy, NSHM Knowledge Campus, Kolkata-Group of Institutions, 124, B L Saha Road, Kolkata, West Bengal, India. \\ c Department of Pharmaceutics, Guru Nanak Institute of Pharmaceutical Science and Technology, 147f, Nilgunj Road, Sahid Colony, Panihati, Kolkata, \\ West Bengal, India. \\ *Corresponding author's E-mail: dipanjanaash16@gmail.com
}

Received: 08-09-2021; Revised: 16-11-2021; Accepted: 24-11-2021; Published on: 15-12-2021.

\section{ABSTRACT}

Organogelators induce 3-D networked structures in apolar solvent molecules via cross-linking of non-covalent self-assembled aggregates below the gelation temperature. The objective of the present investigation was to evaluate the effects of different Span 40 concentrations on gelation kinetics and drug release behaviour of topical soybean oleogels. An inversely proportional relationship was observed between gelation time, melt flow index and concentration of Span 40 in soybean oleogels. Gompertz model was employed on gelation kinetics data to determine organogelator and oil parameters which are assumed to be associated with thermal stability and gel flexibility respectively. Formulation OGS2 (18\% W/V Span 40 ) formed less viscous, thermally stable and presumably more flexible oleogel compared to other formulations. Nearly ideal zero-order release of paracetamol was achieved from OGS*2 following Fickian diffusion. However, slow drug release profiles, higher t50 values were observed with oleogels having $20-24 \% \mathrm{w} / \mathrm{v}$ Span 40 which followed Korsmeyer-Peppas kinetics with non-Fickian diffusion.

Keywords: Gel flexibility, Gelation kinetics, Gompertz model, Melt flow index, Soybean oleogels, Span 40.

QUICK RESPONSE CODE $\rightarrow$

DOI:

10.47583/ijpsrr.2021.v71i02.013

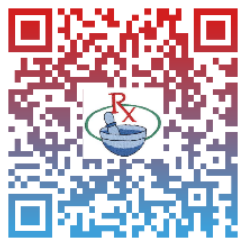

DOI link: http://dx.doi.org/10.47583/ijpsrr.2021.v71i02.013

\section{INTRODUCTION}

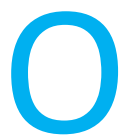

rganogelators which are low molecular weight viscoelastic materials, promote non-covalent selfassembly of various types of aggregates, such as fibres, strands, and tapes, which are cross-linked among themselves through "junction zones" resulting in the formation of 3-D networked structures in the solvent molecules leading to oleogels below the gelation temperature $\left(T_{\mathrm{g}}\right)^{1}{ }^{1}$ Sterol, sorbitan monostearate (Span 60), sorbitan mono palmitate (Span 40), lecithin and cholesteryl anthraquinone derivatives have been used as organogelators at different concentrations in the development of oleogels with different apolar organic solvents such as cyclo-hexane, benzene and carbon tetrachloride, vegetable oils (sunflower oil, rice bran oil, sesame oil, olive oil, mustard oil etc.) and mineral oil. ${ }^{2-5}$ For each apolar solvent-organogelator combination, there exists a unique critical gelator concentration (CGC), i.e. minimum concentration of gelator necessary to induce gelation of the particular solvent/oil. ${ }^{4}$

An ideal oleogel for the topical application would be one with good organoleptic properties, satisfactory extrudability and spreadability, pseudo-plastic flow behaviour, high flexibility, high thermal stability and improved drug release. The properties of oleogels are governed by relatively weak and non-bonded interactions such as hydrogen bonding, Vander Waals interactions and $\pi$-stacking of gelators. ${ }^{6}$

The nourishing property of vegetable oils increases their utilization as suitable solvents in topical formulations. It is reported that soybean oil based gels and lotions protect our skin from UVB rays, free radical-induced inflammation, reduce transdermal water loss on the skin and promote skin barrier recovery. ${ }^{7}$

The objective of the present study is to develop paracetamol (PCM) loaded novel soybean oleogels for topical application by varying Span 40 concentration and to evaluate the effects of different organogelator concentrations on in-vitro characteristics of soybean oleogels and establish a relationship between soybean oil parameter and gel flexibility from gelation kinetics study as well as to ascertain the effect of organogelator concentration on drug release behaviour from the oleogels.

\section{MATERIALS}

Soybean oil pouches of same batch (Emami Ltd., India) were procured from the local market, Kolkata, West Bengal in order to maintain a strict control on oil quality. Span 40 was of AR grade and obtained from Loba Chemie and paracetamol IP (PCM) was received as a gift sample from the enlisted vendor. Double-distilled water was used throughout the study, wherever required. For 
hemocompatibility study, fresh goat blood was collected in the heparin-coated tube and stored at $-4^{\circ} \mathrm{C}$.

\section{METHOD}

Accurately weighed paracetamol $(2 \% \mathrm{w} / \mathrm{v}$ for drug-loaded batches) and Span 40 were dissolved in soybean oil maintained at $60^{\circ} \mathrm{C}$ with continuous stirring in mechanical stirrer (REMI) at 500 r.p.m for $1 \mathrm{~h}$ after which a clear, homogeneous solution was obtained and it was allowed to cool down subsequently to $25^{\circ} \mathrm{C}$ to form oleogels having different concentration $(\% \mathrm{w} / \mathrm{v})$ of organogelator. Composition of the various batches of oleogel formulations is presented in Table 1.

Table 1: Composition of Soybean Oil Based Oleogels(\%w/v)

\begin{tabular}{|c|c|c|c|}
\hline Batch & PCM & Span $\mathbf{4 0}$ & Soybean oil \\
\hline OGS* 1 & 2 & 16 & 82 \\
\hline OGS* 2 & 2 & 18 & 80 \\
\hline OGS* 3 & 2 & 20 & 78 \\
\hline OGS* 4 & 2 & 22 & 76 \\
\hline OGS* 5 & 2 & 24 & 74 \\
\hline
\end{tabular}

OGS are the blank gels of the corresponding drug-loaded gels

\section{Compatibility study}

\section{Fourier transformed infrared (FT-IR) spectroscopy}

Infrared spectroscopic studies of oleogel components and trial batch of the drug-loaded formulation (OGS* 2) were carried out by FT-IR spectroscopy (Bruker, Alpha-T, Germany) in attenuated total reflectance (ATR) mode in the range of $4000-500 \mathrm{~cm}^{-1}$.

\section{Physico-chemical characterisation}

\section{Organoleptic evaluation}

The freshly prepared oleogels were subjected to organoleptic evaluation for their color, odor, opacity and appearance.

\section{Drug content study 8}

A definite amount of drug-loaded oleogel was mixed with phosphate buffer ( $\mathrm{pH} \mathrm{5.8)} \mathrm{to} \mathrm{obtain} \mathrm{uniform} \mathrm{dispersion}$ that was kept undisturbed for $48 \mathrm{hr}$. The dispersion was filtered through Whatman filter paper (No.1). An aliquot of filtrate was suitably diluted and analysed by UV-visible spectrophotometer (UV 1800 UV-vis spectrophotometer, Shimadzu Corporation) at a wavelength of $249 \mathrm{~nm}$.

\section{Rheological study ${ }^{9}$}

Rheological behaviour of oleogels was studied by using Brookfield digital viscometer (Model LVDVI+) at 25으 by varying shear rate from 1-5 r.p.m (spindle 6) for $1 \mathrm{~min}$ each. Ostwald de-wale Power model (equation 1) was employed for modelling of viscosity for the determination of flow consistency index (k) and flow behaviour index (n) from the relationship between shear stress $(T)$ and shear rate $(\gamma)$.

$$
T=k^{*} \gamma^{n}
$$

\section{Thermal analysis}

\section{Gelation kinetics study}

Gelation kinetics study of oleogels was performed by nepheloturbidometry (ELICO ${ }^{\circ} \mathrm{CL} 52 \mathrm{D}$, ELICO India). Oleogel in sol state was transferred to Nessler cylinder and the light was allowed to pass through the turbid sample where suspended particles scattered light. Transformation of sol to gel was characterised by increase in turbidity which continued for a certain period of time after which there was no further increase in turbidity. The time at which turbidity attained a constant value is defined as gelation time. The turbidity intensity was measured at $20 \mathrm{~s}$ interval and expressed in terms of nepheloturbidity unit (NTU).

\section{Gelation kinetics modelling ${ }^{10}$}

Gompertz model was employed for modelling of gelation kinetics. This non-linear model (equation 2) indicates a relationship between turbidity intensity (NTU) (Y), concentration of gelator in $\% \mathrm{w} / \mathrm{v}(\rho)$ and time for gelation in $h(x)$.

$\alpha$ is defined as non-polar solvent(oil) parameter and is related to gel flexibility whereas $\beta$ indicates organogelator parameter and can be related to gel's thermal stability.

$$
\log Y=\alpha+b \rho^{x}
$$

In the above equation, $\rho^{x}$ is defined as

$$
\rho^{x}=\rho_{1}^{x}+\rho_{2}^{x}+\rho_{3}^{x}+\ldots
$$

Where $\rho_{1}$ indicates organogelator concentration, $\rho_{2}$ indicates surfactant concentration; $\rho_{3}$ indicates other additive concentration and so on.

\section{Determination of gel-sol transition temperature $\left(T_{g}\right)^{4}$}

Thermal analysis of oleogel was done by drop ball method for determination of the gel-sol transition temperature $\left(T_{g}\right)$. A stainless steel ball having the diameter of $1 / 8^{\text {th }}$ inch and weight of $230 \mathrm{mg}$ was placed over the formulation in a beaker and attached with a melting point apparatus (EI931). The formulation was heated at a rate of $10 \mathrm{C} / \mathrm{min}$. The temperature at which the ball started to move into the gel was noted and considered as the gel-sol transition temperature of the oleogel $\left(T_{g}\right)$.

\section{Melt flow index ${ }^{11}$}

Melt flow index (MFI) is defined as the ease of melt flow of thermoplastic material in gram over a period of $10 \mathrm{~min}$ at a certain standard temperature (i.e, $65^{\circ} \mathrm{C}$ for gel formulations) when checked in melt flow tester. A fixed weight of oleogel (10 g) was poured into the cylinder and temperature was set at $65^{\circ} \mathrm{C}$ to prevent thermal degradation of gel at higher temperature as specified in 
reported method. Pressure was applied with the piston bar to the cylinder using $10 \mathrm{~g}$ of weight above the piston bar after setting at the predefined temperature. Sample melt flow occurred through the die face in the form of wire and was collected after $10 \mathrm{~min}$ and was further subjected to weighing to determine its mass.

\section{Drug diffusion studies}

\section{In-vitro drug diffusion study and modelling of release} kinetics ${ }^{4}$

Modified Franz diffusion cell was used to perform the in vitro drug release study on prepared oleogels through dialysis membrane-60 (HIMEDIA LA 330-5MT). Accurately weighed drug-loaded oleogel containing PCM equivalent to $4 \mathrm{mg}$ was placed on the membrane and wetted slightly with phosphate buffer ( $\mathrm{pH} 5.8)$. The buffer solution in the receptor compartment was maintained at $32 \pm 0.5 \circ \mathrm{C}$. An aliquot of $1 \mathrm{~mL}$ was withdrawn every hour for $7 \mathrm{~h}$ and replenished with fresh buffer. Following appropriate dilution, aliquot was analysed by UV-visible spectrophotometer (UV 1800 UV-vis spectrophotometer, Shimadzu Corporation) at a wavelength of $249 \mathrm{~nm}$. The study was performed in triplicate. From drug release profile the time taken for diffusion of $50 \%$ drug i.e., $t_{50}$ value was calculated for all gel formulations for modelindependent comparison. Simultaneously, the drug release data of oleogels were subjected to kinetic modelling to determine the drug release pattern and the diffusion mechanism.

\section{Steady-state flux, permeability co-efficient determination $^{12}$}

The measurement of flux across human skin provides a valuable insight into the formulation development of any dermatological product. The steady-state flux of PCM from oleogels across the artificial dialysis membrane is defined as follows.

$$
S S_{f l u x}=d Q / d t^{*} 1 / A
$$

Where,

$\mathrm{SS}_{\text {flux }}=$ steady-state flux of drug $\left(\mathrm{mg} / \mathrm{cm}^{2} \cdot \mathrm{hr}\right) ; \mathrm{dQ} / \mathrm{dt}=$ slope of the linear portion of the curve i.e. cumulative amount per unit time $(\mathrm{mg} / \mathrm{hr}) ; \mathrm{A}=$ diffusional area $\left(\mathrm{cm}^{2}\right)$

Permeability co-efficient $\left(K_{p}\right)$ is quantified by the following equation.

$$
K_{p}=S S_{f l u x} / C_{a p p}
$$

Where,

$\mathrm{C}_{\mathrm{app}}=$ initial concentration of the drug in the gel formulation. In the present study, it was expressed as
$\% w / v$ i.e. weight of drug actually present in the volume of gels taken for permeation study.

\section{Accelerated stability study ${ }^{13}$}

Accelerated stability study includes thermo-cycling or freeze/thaw cycling, and syneresis measurements that help to predict the effect of temperature change on stability of anhydrous organogels. The purpose of the study was to assess the change in gelation time with consecutive freeze-thaw cycles. Thermo-cycling or freezethaw cycling method involves incubation of the freshly prepared gel samples at 65 으 for 15 mins till the formation of sol state followed by gelation when time was noted. Then these oleogels were incubated at 4 으 for $15 \mathrm{~min}$ after which stored at $25 \circ \mathrm{C}$ for $48 \mathrm{hr}$. The process was repeated for 6 cycles for oleogel and at the end of each cycle, gelation time was noted.

\section{RESULTS AND DISCUSSION}

\section{Oleogel formation}

Oleogel formation for soybean oil occurred at all the selected Span concentrations. The dissolution of Span 40 in the soybean oil at $60^{\circ} \mathrm{C}$ resulted in the formation of clear homogeneous solution but change in solubility parameter of Span 40 molecules with lowering of temperature to $25^{\circ} \mathrm{C}$ decreased the affinity between continuous oil phase (soybean oil) and Span and therefore a three dimensional self-assembled structure of gelator was formed by capturing oil molecules within. ${ }^{4}$

\section{Fourier transformed (FT-IR) Spectroscopy}

FT-IR study was performed for Span 40, soybean oil, PCM, drug-loaded oleogel (OGS* 2) [Fig.1] Peaks at 2913, 2855, 1744,1157 and $719 \mathrm{~cm}^{-1}$ were found in the spectrum of soybean oil. Most of the characteristic peaks of Span 40, soybean oil and drug were visible in drug-loaded oleogel. FTIR analysis of the oleogels revealed slight shifting in the peaks of the individual components as well as minor changes in peak intensity indicating otherwise compatibility between the oil, organogelator and the drug. Absence of the broad peak at $3300 \mathrm{~cm}^{-1}$ in oleogels indicated intermolecular hydrogen bonding among the fatty acyl groups of gelator and oil molecules which is responsible for imparting strength to the gels. ${ }^{14-15}$

\section{Physico-chemical characterisation of oleogels}

The organoleptic properties of prepared blank oleogels are summarized in Table 2 . The drug content study revealed that all formulations contained drug in the range of 9296\%. (Table 2) 


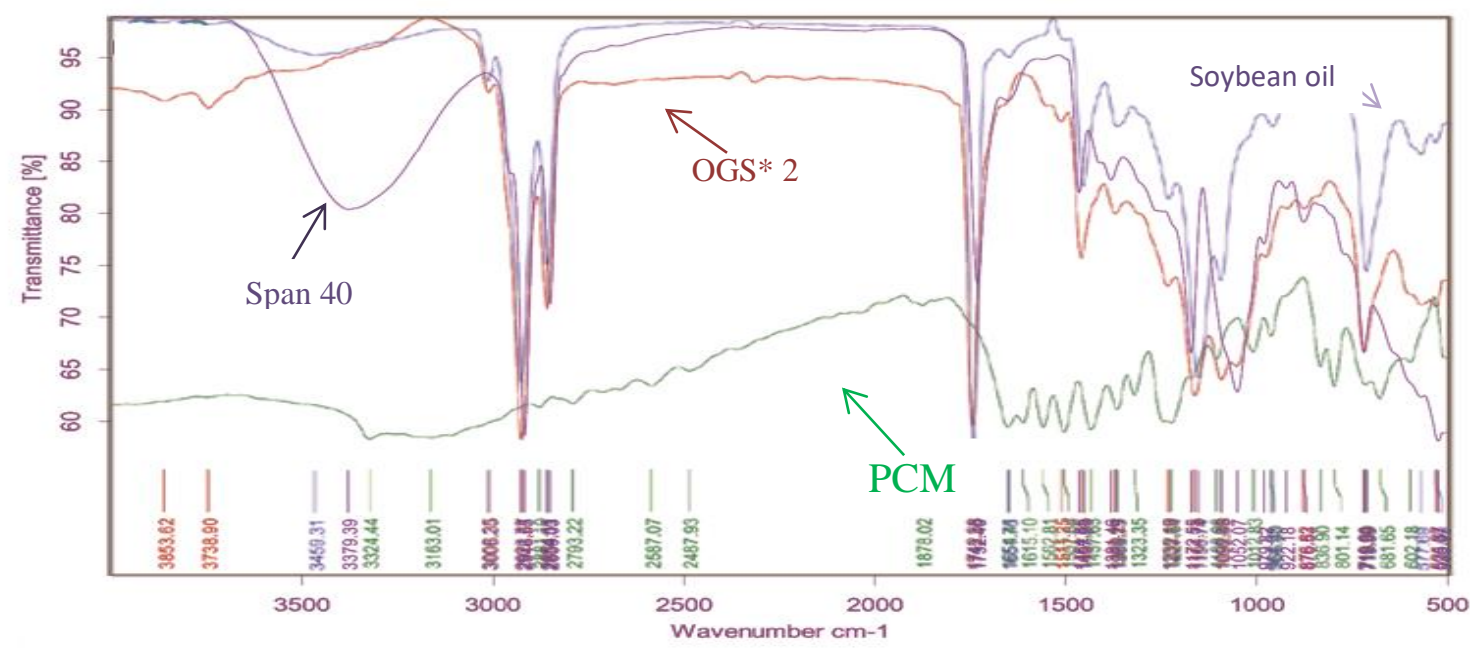

Figure 1: FT-IR Spectra Of Drug-Loaded Oleogel Of Soybean Oil (Ogs*2) Along With Raw Materials

Table 2: Organoleptic Characteristics and Drug Content Assay of Soybean Oleogels

\begin{tabular}{|c|c|c|c|c|c|}
\hline \multirow{2}{*}{ Batch } & \multicolumn{4}{|c|}{ Organoleptic characteristics } & \multirow{2}{*}{ Drug content $^{+}(\%)$} \\
\hline & Colour & Odour & Opacity & Appearance & \\
\hline OGS2 & Yellowish-white & Odourless & Opaque & Smooth-oily & $96 \pm 0.2$ \\
\hline OGS 3 & Yellowish- white & Odourless & Opaque & Smooth-oily & $95 \pm 0.8$ \\
\hline OGS 4 & Yellowish- white & Odourless & Opaque & Smooth-oily & $93 \pm 0.4$ \\
\hline OGS 5 & Yellowish- white & Odourless & Opaque & Smooth-oily & $92 \pm 0.8$ \\
\hline
\end{tabular}

\section{Rheological study}

The order of viscosity for soybean oil based blank oleogels is as follows:

OGS5> OGS4> OGS3> OGS2 [Fig. 2].

Modelling of viscosity data revealed all the formulations to exhibit non-Newtonian pseudo plastic flow behaviour.

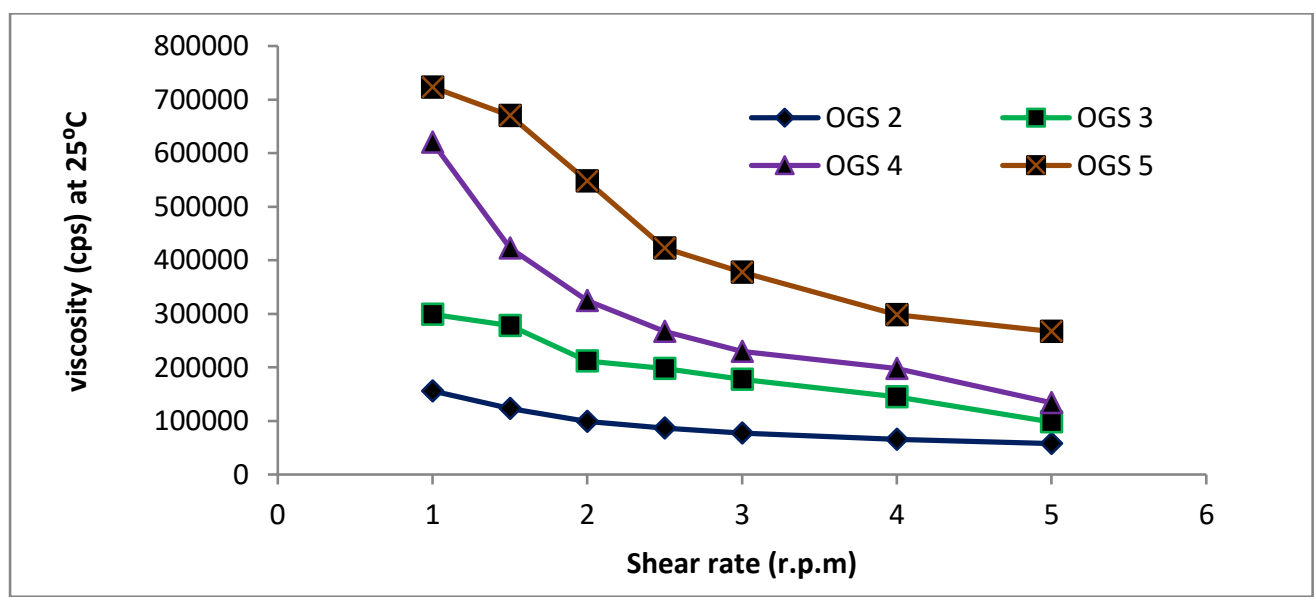

Figure 2: Effect of Organogelator Concentration on Rheological Behaviour of Soybean Oleogels

\section{Thermal analysis}

The gelation kinetics was studied by monitoring the change in turbidity during cooling of the blank oleogels. The change in gelation time with gelator concentration is shown in Table 3. Gompertz model was employed for modelling of gelation kinetics to determine soybean oil parameter $(\alpha)$ and organogelator parameter $(\beta)$ [Table 3] which are related to gel flexibility and thermal stability of the oleogels respectively. Gel-sol transition temperature 
( $T_{\mathrm{g}}$ ) of the blank oleogels is presented in Table 3. $T_{\mathrm{g}}$ value was found to increase with increase in gelator concentration.

The order of MFI of soybean based oleogels is presented as follows:

\section{OGS $2>0 G S 3>0 G S 4>0 G S 5$}

Thermal analysis of oleogels suggested that increase in gelator concentration decreased gelation time and caused gel-sol transformation to occur at a higher temperature. The $T_{g}$ values of the formulations were lower than the melting point of Span 40. The increase of $T_{g}$ with the increase in gelator concentration indicated that selfassembly in the gel state is governed by strong intermolecular interactions attributable to Span 40.Gel-sol transformation is a reversible process as the gels reformed on cooling exhibiting same properties as before $\left({ }^{16}\right.$
Oleogel, OGS 2 was found to possess higher $\alpha$ - value compared to other formulations indicating higher flexibility. Moreover, OGS 2 was found to possess lower viscosity than other Span-based formulations suggesting ability of lower concentration of Span 40 to result in flexible (higher $\alpha$-value) structure. Higher viscosity and lower $\alpha$-value of OGS 5 suggested the formation of a more compact mesh-like self-assembled structure within the oleogel. Better thermal stability of OGS 5 was established from the lower $\beta$-value and simultaneously higher $T_{\mathrm{g}}$ value. As the concentration of gelator was increased, $\alpha$-value decreased indicating the formation of comparatively rigid gels with higher viscosity and better thermal stability as manifested in increasing $\beta$-value and higher $T_{g}$. The oleogel (OGS 2 ) with higher $\alpha$-value and low viscosity is expected to demonstrate improved drug release owing to higher gel flexibility.

Table 3: Thermal Analysis Data of Soybean Oil Based Oleogels

\begin{tabular}{|c|c|c|c|c|c|}
\hline Batch & $\begin{array}{c}\text { Gel-sol transition } \\
\text { temperature }\left(\mathbf{T}_{\mathbf{g}} \mathbf{)}(\mathbf{-} \mathbf{C})^{+}\right.\end{array}$ & $\begin{array}{c}\text { Gelation time } \\
(\mathbf{s e c})^{+}\end{array}$ & $\begin{array}{c}\text { Melt flow index } \\
(\mathbf{g m} / \mathbf{1 0 s e c})^{+}\end{array}$ & \multicolumn{2}{|c|}{ Modelling of gelation kinetics } \\
\hline OGS 2 & $42 \pm 0.3$ & $1060 \pm 1.2$ & $0.87 \pm 0.7$ & 2.0726 & 0.5791 \\
\hline OGS 3 & $46 \pm 0.5$ & $460 \pm 1.3$ & $0.56 \pm 0.3$ & 2.062 & 0.8454 \\
\hline OGS 4 & $48 \pm 0.4$ & $320 \pm 1.7$ & $0.45 \pm 0.2$ & 2.054 & 0.9234 \\
\hline OGS 5 & $49 \pm 0.5$ & $260 \pm 1.5$ & $0.34 \pm 0.6$ & 2.034 & 0.9985 \\
\hline
\end{tabular}

\section{Drug diffusion studies}

The drug release profiles of PCM from the prepared oleogels are presented in Fig. 3. The percentage of drug release decreased with increase in Span 40 concentration. Results of kinetic modelling of drug release studies are tabulated in Table 4. Solubility and partition co-efficient of PCM play a significant role on release of PCM from soybean based oleogels. ${ }^{17}$ Improved drug release from oleogel, OGS* 2 is attributed to the flexible gel network as evident from the thermal analysis. Satapathy reported earlier that the release of the drug molecules from mustard oil and ground nut oil based organogels decreased with increase in gelator concentration due to the formation of dense network structures that hindered drug diffusion. ${ }^{5}$ It was found that OGS* 2 followed zero order release kinetics with Fickian diffusion, but drug release from other formulations occurred via non-Fickian diffusion and followed Korsmeyer-Peppas model. Moreover, constant increase in the values of $t_{50}$, and decreasing $\mathrm{SS}_{\text {flux }}$ and permeability coefficients were observed in gels with higher concentration of Span 40. Data from in vitro drug diffusion studies indicated negative impact of increasing concentration of organogelator on drug release behaviour from thermally stable oleogels.

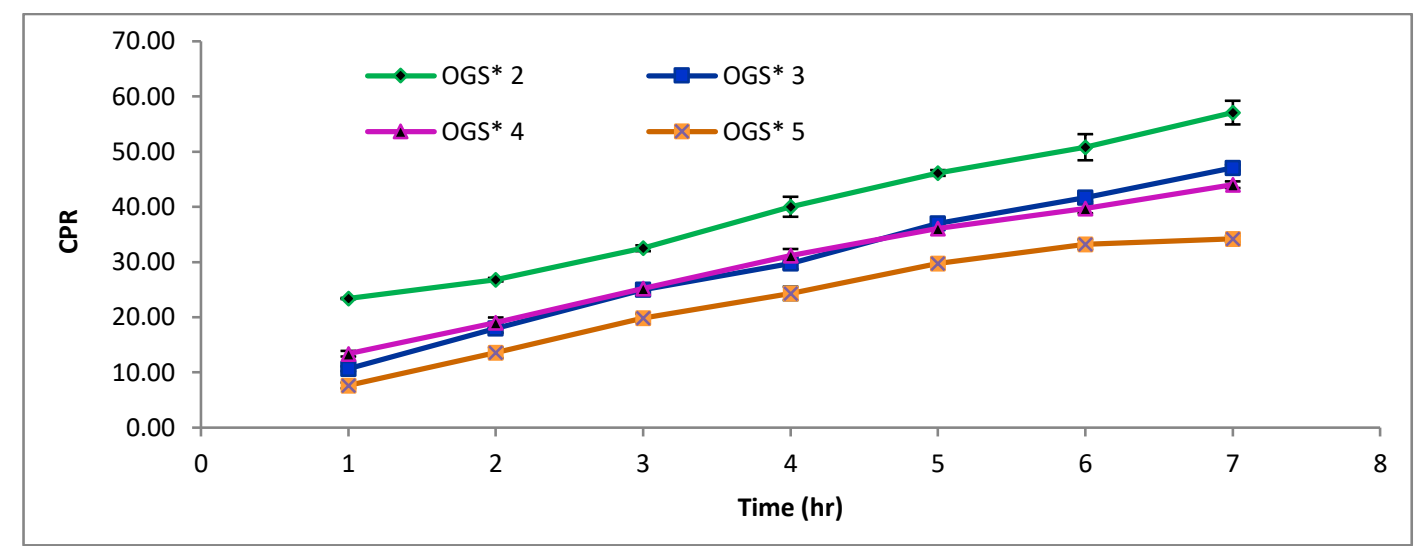

Figure 3: Effect of Span 40 Concentrations on Drug Release Profiles from Soybean Oleogels. Error bars represent mean \pm sandard deviation for 3 experiments $(n=3)$ 
Table 4: Kinetic Modelling Of Drug Release Data And Determination Of Drug Diffusion Parameters From Oleogels Of Soybean Oil

\begin{tabular}{|r|c|c|c|c|c|}
\hline Batch & $\begin{array}{c}\text { Kinetics } \\
\text { followed }\end{array}$ & $\begin{array}{c}\text { Mechanism of } \\
\text { diffusion }\end{array}$ & $\mathbf{t}_{50}(\mathbf{h r})^{+}$ & $\mathbf{S S}_{\text {flux }}\left(\mathbf{m g} / \mathbf{c m}^{2} / \mathbf{h r}^{+}\right.$ & $\mathbf{K}_{\mathbf{p}}\left(\mathbf{c m}^{2} / \mathbf{h r}^{+}\right.$ \\
\hline OGS 2* & Zero & Fickian & $6.0 \pm 0.2$ & $2.69 \pm 0.6$ & $1.34 \pm 0.2$ \\
\hline OGS 3* & $\begin{array}{c}\text { Korsmeyer- } \\
\text { Peppas }\end{array}$ & Non-Fickian & $7.4 \pm 0.3$ & $1.98 \pm 0.4$ & $0.99 \pm 0.3$ \\
\hline OGS 4* & $\begin{array}{c}\text { Korsmeyer- } \\
\text { Peppas }\end{array}$ & Non-Fickian & $8.0 \pm 0.3$ & $1.68 \pm 0.5$ & $0.84 \pm 0.4$ \\
\hline OGS 5* & $\begin{array}{c}\text { Korsmeyer- } \\
\text { Peppas }\end{array}$ & Non-Fickian & $8.5 \pm 0.4$ & $0.87 \pm 0.3$ & $0.44 \pm 0.5$ \\
\hline
\end{tabular}

\section{Accelerated stability study}

Soybean oil based oleogels were found to be stable up to 10 cycles. [Fig. 4] (only 6 cycles are reported here). Accelerated stability studies on oleogels showed them to be stable with slight increase in gelation time for OGS3, OGS 4 and OGS5 from $2^{\text {nd }}$ freeze/thaw cycle. No change in gelation time was seen with OGS 2 even after 6 cycles indicating the oleogel structure to be able to withstand effects of temperature change.

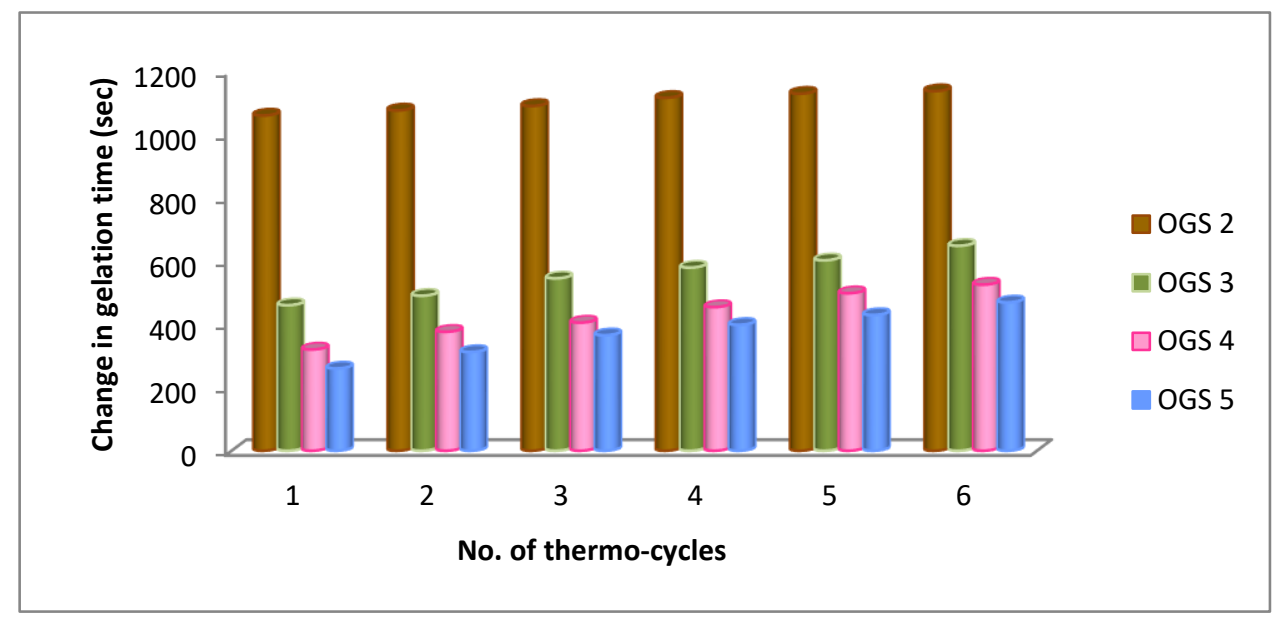

Figure 4: Change in Gelation Time of Oleogels of Soybean Oil on Exposure to 6 FreezeThaw Cycles

Combining the results from the above studies, it can be concluded that increase in concentration of Span 40 as organogelator produced most dense soybean oleogel with higher viscosity, high gel-sol transition temperature, and higher thermal stability but with remarkable decrease in drug diffusion. But, soybean oleogel with $18 \%$ w/v Span 40 produced a stable topical formulation with satisfactory organoleptic, thermal and rheological properties and zeroorder drug release profile, ideally suitable for controlled release applications.

\section{CONCLUSION}

Soybean oleogels of varying thermal and rheological properties with different drug release profiles and kinetics were obtained by changing the concentration of Span 40. Increase in organogelator concentration improved the thermal stability of oleogels but produced rigid network with very low drug release profiles and steady state fluxes.
Acknowledgments: Authors contributed equally to the design and implementation of the research, to the analysis of the results and the writing of the manuscript.

\section{REFERENCES}

1. Sahoo S, Kumar N, Bhattacharya C, Sagiri SS, Jain K, Pal K, Ray SS, Nayak B. "Organogels: Properties and applications in drug delivery". Des Monomers Polym. 2011; 14: 95-108.

2. Mujawar NK, Ghatage SL, Yeligar VC. "Organogel: Factors and its importance". Int J Pharm Chem Biol Sci. 2014; 4: 758-73.

3. Singh VK, Pramanik K, Ray SS, Pal K. "Development and characterization of sorbitan monostearate and sesame oil-based organogels for topical delivery of 
antimicrobials". AAPS Pharm Sci Tech. 2015; 16: 293305.

4. Shah DK, Sagiri SS, Behera B, Pal K, Pramanik K. "Development of olive oil-based organogels using sorbitan monopalmitate and sorbitan monostearate: a comparative study". J Appl Poly Sci. 2013; 129: 793804.

5. Satapathy D, Sagiri SS, Pal K, Pramanik K. "Development of mustard oil-and groundnut oil-based Span 40 organogels as matrices for controlled drug delivery". Des Monomers Polym. 2014; 17: 545-56.

6. Suzuki M, Nakajima $Y$, Yumoto $M$, Kimura M, Shirai H. Hanabusa K. "Effects of hydrogen bonding and van der waals interactions on organogelation using designed low-molecular-weight gelators and gel formation at room temperature". Langmuir. 2003; 19: 8622-4.

7. Lin TK, Zhong L, Santiago JL. “Anti-inflammatory and skin barrier repair effects of topical application of some plant oils". Int J Mol Sci. 2018; 19: 1-21.

8. Balata GF, Shamardl HAEM.. "Formulation of cholorpheniramine maleate in Span 60/ Tween 20 based organogels for transdermal delivery". J Inno Pharm Bio Sci. 2017; 4: 49-57.

9. Singh VK, Anis, A, Zahrani SMA, Pradhan DK, Pal K. "FTIR, electrochemical impedance and iontophoretic delivery analysis of guar gum and sesame oil based bigels". Int J Electrochem Sci. 2014; 9: 5640-50.
10. Shukla AK, Yadav SK, Tiwari V. "Linear models for Sshaped growth curves". J Stat App Prob. 2015; 4:113-7.

11. Singh R, Kumar R. "Friction welding of dissimilar plasticbased material by metal powder reinforcement". 2017; in Hashmi MSJ (Ed.) Reference module in materials science and materials engineering, Dublin, Ireland; pp. 1-16.

12. Bartosova L, Bajgar J. "Transdermal drug delivery in vitro diffusion cells". Curr Med Chem. 2012; 19: 4671-7.

13. Singh VK, Behera B, Sagiri SS, Pal K, Anis A, Bhattacharya MK. "Organogels in controlled drug delivery", 2015; in Mishra M (Ed.) Handbook of encapsulation and controlled release, London, UK: CRC Press; p. 1035-66.

14. Dautel OJ, Robitzer M, Lere Porte JP, Serein Spirau F, Moreau JJ. "Self-organized ureido substituted diacetylenic organogel. Photo polymerization of onedimensional supramolecular assemblies to give conjugated nanofibers". J Am Chem Soc. 2006; 128: 16213-23.

15. Li Y, Wang $T$, Liu M. "Ultrasound induced formation of organogel from a glutamic Ddendron". Tetrahedron. 2007; 63: 7468-73.

16. Murdan S, Gregoriadis G, Florence AT. "Interaction of a nonionic surfactant-based organogel with aqueous media". Int J Pharm. 1999; 180: 211-4.

17. Gupta R, Kumar M, Sharma HK. "Span 80-Tween 80 based fluid-filled organogel for topical delivery of fluconazole". Int J Sci Res Rev. 2014; 3:29-46.

Source of Support: The author(s) received no financial support for the research, authorship, and/or publication of this article.

Conflict of Interest: The author(s) declared no potential conflicts of interest with respect to the research, authorship, and/or publication of this article.

For any question relates to this article, please reach us at: editor@globalresearchonline.net New manuscripts for publication can be submitted at: submit@globalresearchonline.net and submit_ijpsrr@rediffmail.com 